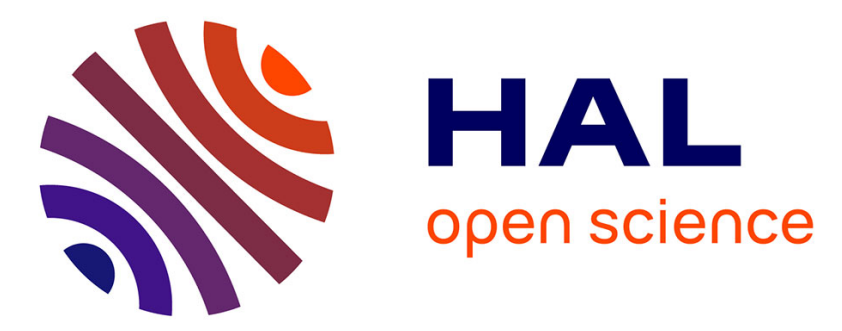

\title{
How to improve CSMA-based MAC protocol for dense RFID reader-to-reader Networks?
}

Ibrahim Amadou, Abdoul Aziz Mbacké, Nathalie Mitton

\section{To cite this version:}

Ibrahim Amadou, Abdoul Aziz Mbacké, Nathalie Mitton. How to improve CSMA-based MAC protocol for dense RFID reader-to-reader Networks?. ADHOC-NOW - 13th International Conference on AdHoc Networks and Wireless, Jun 2014, Benidorm, Spain. hal-00969117

\section{HAL Id: hal-00969117 https://hal.inria.fr/hal-00969117}

Submitted on 2 Apr 2014

HAL is a multi-disciplinary open access archive for the deposit and dissemination of scientific research documents, whether they are published or not. The documents may come from teaching and research institutions in France or abroad, or from public or private research centers.
L'archive ouverte pluridisciplinaire HAL, est destinée au dépôt et à la diffusion de documents scientifiques de niveau recherche, publiés ou non, émanant des établissements d'enseignement et de recherche français ou étrangers, des laboratoires publics ou privés. 


\title{
How to improve CSMA-based MAC protocol for dense RFID reader-to-reader Networks?
}

\author{
Ibrahim Amadou, Abdoul A. Mbacké, Nathalie Mitton \\ Inria Lille - Nord Europe - France \\ Email: firstname.lastname@inria.fr
}

\begin{abstract}
Due to the dedicated short range communication feature of passive radio frequency identification (RFID) and the closest proximity operation of both tags and readers in a large-scale dynamic RFID system, when nearby readers simultaneously try to communicate with tags located within their interrogation range, serious interference problems may occur. Such interferences may cause signal collisions that lead to the reading throughput barrier and degrade the system performance. Although many efforts have been done to maximize the throughput by proposing protocols such as NFRA or more recently GDRA, which is compliant with the EPCglobal and ETSI EN 302208 standards. However, the above protocols are based on unrealistic assumptions or require additional components with more control packet and perform worse in terms of collisions and latency, etc. In this paper, we explore the use of some well-known Carrier Sense Multiple Access (CSMA) backoff algorithms to improve the existing CSMA-based reader-to-reader anti-collision protocol in dense RFID networks. Moreover, the proposals are compliant with the existing standards. We conduct extensive simulations and compare their performance with the well-known state-of-the-art protocols to show their performance under various criteria. We find that the proposals improvement are highly suitable for maximizing the throughput, efficiency and for minimizing both the collisions and coverage latency in dense RFID Systems.
\end{abstract}

\section{Introduction}

Most radio frequency identification applications, such as supply markets, localisation and objects tracking, activity monitoring and access control and security, etc., use passive RFID tags, which communicate with the RFID reader by modulating its reflection coefficient (backward link) to incoming modulated RF signal from the reader (forward link). However, unlike the traditional radio communication systems, in such systems, the RF signal does not provide reciprocity between forward and backward links because the reflected RF signal from a tag is inversely proportional to the fourth power of the distance between reader and tag [18]. For example, in the European Regulation [20], the reader output power of 2 Watt Effective Radiated Power (ERP) limits the reader-to-tags read range to a maximum distance of 10 meters while reader-to-reader interference range may 
reach 1000 meters [19]. This link unbalance requires in above large-scale applications of RFID systems to deploy a large number of RFID readers allowing the coverage of the interested environment. A direct consequence of this feature deployment is the operation within the closest proximity of several tens or hundreds of readers in order to overcome the shortcoming of the backward communication distance. However, due to readers close proximity, when nearby readers simultaneously try to communicate with tags located within their interrogation range, serious interference problems may occur. This is mainly due to the overlapping of readers' field. Such interferences may cause signal collisions that lead to the reading throughput barrier and degrade the system performance. Although collision problems can also appear during the tag communication [12], [4] most of these problems are considered to be solved and are part of patents developed by EPCglobal standard [4]. While the reader-to-reader collision (RRC) problem obtained few attention because previous applications of RFID systems considered only a reader with several tags, the design of an efficient reader-to-reader anti-collision protocol has emerged as the most interesting research issues in recent years. The key to make the RFID system efficient is to schedule readers activities so that neither interferences nor collisions may occur.

The state-of-the-art protocols can be broadly classified as CSMA-based $[2,11$, $20]$ and activity scheduling based $[1,3,6,7,15]$ through time division, frequency or by putting together both approaches. The former approach is considered as an efficient and more adaptive approach in large-scale RFID reader networks because it is full-distributed algorithm and it does need neither synchronization nor additional resource (e.g. server) like in the latter approach. However, the existing protocols still suffer from traditional backoff scheme in dense RFID networks as it is recently observed in NFRA [3] and GDRA [1]. Therefore, the backoff algorithm for CSMA-based may need to be investigated in depth in order to maximize the network throughput but also to minimize the collisions which still exist as they observed in [1].

In this work, we investigate the use of an adaptation of some well-known adaptive backoff algorithms proposed in mobile ad hoc networks (MANETs) in order to improve the performance of CSMA-based anti-collision protocol for dense RFID networks. Our objective is to use protocols such as Idle Sense [9], Sift [14] and Reverse Backoff [13] to perform channel access through broadcasting message with almost zero-collision or in collision-free fashion. Once the channel is reserved by a reader, it can communicate with tags located in its interrogation area in collision-free fashion. Furthermore, our aim is, based on observed characteristics of above protocols, to propose a novel medium access control protocol which can significantly improve the performance metrics such as throughput, fairness behavior during channel access and with the minimum latency in large-scale RFID system. Unlike the majority of previous works, in this work, we extend our performance evaluation in terms of qualitative and quantitative criteria.

The rest of this paper is organized as follows. Section 2 introduces the system model. Section 3 reviews the state-of-the-art anti-collision protocols for RFID 
networks. In Section 4, we provide a general overview of the basic adaptive backoff algorithms of MANETs investigated in this work in order to improve the performance of CSMA-based reader-to-reader anti-collision protocols. In Section 5, we investigate their performance via extensive simulation under various RFID environment scenarios and according to several criteria. Finally, Section 6 concludes by discussing future research direction.

\section{System Model}

We consider a large-scale RFID system with multiple readers and homogeneous local density of RFID tags within the interrogation area. The readers are randomly deployed with uniform distribution on 2D plane. Readers are assumed to have homogeneous properties. Therefore, their communication range is assumed to be the same. Similar to ETSI EN 302 208-1 regulation [20], in this paper, we assume the use of multichannel network scheme. We assume the existing of an overlapping area in their interrogation areas. Note that the knowledge of these overlapping areas is not a necessity in this work. We assume that readers are able to accurately estimate their neighborhood reader size in the case of adaptive backoff schemes that are based on the number of active readers. Note also that this assumption can be easily removed because, as in [1], we also make use of two bistatic antennas.

But in the future, our investigation will focus on designing a novel full distributed medium access control protocol which can not involve any additional requirement such as bistatic antennas. Therefore, in the next step of this study, according to our target objective in terms of performance metrics and the selected approach, we will either investigate the design of an efficient neighbor estimation mechanism or the adaptive approach with similar performance. Furthermore, it should be able to deal with mobility of both readers and tags in RFID environment.

\section{Related work}

In this section, we present the main characteristics of the state-of-the-art proposed reader-to-reader anti-collision protocols in RFID environment. According to the taxonomy previously introduced, we first overview the CSMA-based approaches. Thereafter, we present the activity scheduling approaches proposed in the literature.

\subsection{CSMA-based approach}

Listen Before TAlK (LBT) is proposed by the European Telecommunication Standard Institute under ETSI EN 302 208-1 [20] for ultra high frequency (UHF) dense RFID environment. With listen before talk concept, each reader must listen for a specified minimum time equal to $5 \mathrm{~ms}$ before confirming that the 
selected channel is not busy. At the end of this fixed period of listening, if the selected channel is unoccupied, reader begins the tags interrogation process, which is called reader-to-tag communication phase, over this channel. Otherwise, it must randomly select a new channel and repeat the same process. However, as the number of readers increases in the system, LBT performs poorly in terms of throughput [1].

PULSE [2] is CSMA-based protocol which is proposed to resolve hidden terminal issue. PULSE proposes the use of two non-interfering channels: control channel and data channel. It consists of periodically sending a beacon on control channel while the reader is communicating with the tags on the data channel. In such a way, the beaconing transmission prevents any another reader to access the channel. This leads to an increase of latency because the reader may need to wait thrice the beaconing transmission before any other channel access attempt. Moreover, its effectiveness in terms of collision avoidance comes at cost of high overhead of periodic beacon, which drastically impacts the energyefficiency behavior when energy is a constraint. Furthermore, the channel access backoff parameter is set without any investigation. This does not mitigate collisions due to the simultaneous channel access which leads to the omission of some tags in the overlapping zone of collide readers. Even if it is presented as a solution to resolve the hidden terminal issue, PULSE does not completely resolve this problem.

Similar to PulSE protocol, DiCA [11] is a distributed CSMA-based protocol with two channels, which aims to provide an energy-efficient collision avoidance protocol. During the channel access process, the reader may not need neither to periodically send beacon message nor to wait additional delay. To completely resolve hidden terminal and exposed terminal problems, DICA adjusts the control channel communication range by doubling the radius from the data channel communication range. As in PULSE protocol, the backoff algorithm is designed without any investigation inducing misreading tags during the reader-to-tags communication.

\subsection{Activity scheduling approach}

Distributed Color Selection (DCS) [15] is one of the first distributed TDMA-based reader-to-reader anti-collision protocols. It is also known as Colorwave in the literature. In DCS, communication is organized in groups of color timeslots, that are called frames or rounds. Each timeslot is composed of two phases: kick and transmission. The kick phase is used by readers that collided in the previous round in order to prevent a new collision in the current frame, while the latter phase is used by readers to read tags located in their interrogation range. Basically, it performs as follows : each reader randomly selects a color timeslot where it can send the request to read tags during its transmission phase. If a collision is observed by colliding readers, they randomly select a new color and reserve them for the next frame. In the next frame, during the kick phase, colliding readers broadcast their color to all their neighborhood in order to prevent a new collision. Based upon information received from collide readers, 
neighborhood readers adjust their color so that a new collision could be avoided. Unfortunately DCS experiences a performance degradation due to the use of a fixed number of colors. Because in a dense network, when the number of colors is too low, DCS experiences many collisions, while, in a low dense network, when the number of used colors is too large, the throughput is degraded because of the a large number of colors. To overcome this inflexibility, authors proposed Variable-Maximum DCS which dynamically adjusts the maximum number of colors regarding the percentage of successful transmissions monitored by the reader in order to improve the performance. A more efficient but centralized version has been proposed in [8].

PROBABILISTIC DSC [7] is an improvement version of DCS which introduces a new parameter $p$, representing the probability to change color after a collision. It is proposed to reduce the number of consecutive collisions due to too many changes in neighborhood of colliding readers. This approach improves DCS. Moreover, PDCS is a multichannel protocol. Distributed Color Noncooperative selection (DCNS) [6] is also TDMA-based protocol, which aims to improve the system throughput even in high density networks.

Neighbor Friendly Reader Anticollision (NFRA) [3] is TDMA-based protocol, which uses a polling server to synchronize and coordinate readers activity. It improves the efficiency of RFID readers networks in term of throughput by allowing readers to operate in collision-free manner during tags reading process. Similar to Colorwave [15], in NFRA, a reader-to-tag communication is scheduled in round by polling server. The round is formed by a fixed number of contention slots, where a reader can contend for reading tags around its communication range, plus a period of time necessary to read tags (e.g. 0.46s). NFRA performs as follows: at the beginning of each round, the server first sends an Arrangement Command $(A C)$, which holds the number of slots (e.g. $\mathrm{MN})$ in the round and advertises also the beginning of round. Upon reception of $A C$, each reader chooses $k$, an integer distributed uniformly in $[1, \mathrm{MN}]$ and waits for receiving the $O C$ from the server, which advertises the slot $k$, before attempting to gain the channel throughout the transmission of beaconing message. If the beaconing is sent without collision, it continues to send an Overriding Frame (OF) message to suppress its active interfering neighbors that have a high value of $\mathrm{k}$. The aims of this suppression mechanism is to ensure that the reader-to-tag communication will operate in collision-free fashion. However, if a collision is observed during the beaconing transmission, the collide readers cancel the transmission scheduling and wait for the next round to attempt. Unfortunately, NFRA implicitly assumes that when a collision occurs, collide readers can detect the collision. While in practice, a collision of broadcasting message can not be detected. NFRA++ [5] aims to improve the fairness of NFRA which performs poorly when the reader neighborhood is too large.

In order to remove unpaired assumption introduced in NFRA, GEOMETRIC Distribution READER Anticollision (GDRA) [19] is proposed with the aims to be compliant with the EPCglobal standard and ETSI EN 302 208-1 regulation and to minimize the reader collision problem by using Sift [14] geometric 
probability distribution function to choose the slot value $k$. To be compliant with ETSI EN 302 208-1 regulation, GDRA is based on multichannel and also proposes to use a $5 \mathrm{~ms}$ as slot size which is the minimum time required to listen the channel before transmission attempting. Instead of assuming the collision detection during the beaconing step, GDRA assumes the use of two bistatic antennas by reader. This avoids the OF transmission. Moreover, it also suppresses the use of $\mathrm{OC}$ by assuming that readers are able to synchronize themselves in a round, which requires additional resources and do not fit dynamic environments.

Unlike CSMA-based protocols, these protocols necessitate a high level of synchronization among the backbone network formed by readers or an additional resource such as centralized server which can address the synchronization problem through wired or wireless connection. In addition to the high level of synchronization requirement, in TDMA-based protocol, the use of dedicated server for explicit coordination restricts the use of these solutions into a limited environment. Moreover, if energy is part of performance requirements, the performance can gradually decrease.

\section{Overview of investigated adaptive protocols}

In this section, we detail the adaptive backoff algorithms taken from wireless MANETs in order to improve existing CSMA-based reader-to-reader anticollision protocols, which still suffer from the backoff scheme. Because the vast majority of observed collisions in RFID system is due to the use of inappropriate backoff scheme. Moreover, how to set the backoff algorithm during channel access in order to make them efficient, however, is far less investigated. [5] has recently proved that by increasing the backoff value of these protocols, they improved the fairness behavior.

So far, many research efforts have been made to improve the performances or the reliability of IEEE $802.11 \mathrm{DCF}$ technique for MANETs by using an adaptive backoff scheme. Here, we look at these well-known solutions that proved their efficiency in high congestion wireless networks. Although there are many full distributed MAC protocols that have been proposed to improve the efficiency of CSMA-based techniques in MANETs, in the following, we mainly focus on particular interest protocols that involve less additional requirements with respect to the RFID reader constraints.

\subsection{Maximum Backoff or Reverse-Backoff}

Generally speaking, the probability of a collision decreases when the contention window size increases. Based on this, [13] introduces reverse backoff (RB). RB starts with a backoff with maximum value decreased every time a beacon expires until some maximum backoff counter, which does not induce the beaconing expiration. It copes with collision problem during the channel access because the backoff counter is large. However, the achieved value depends on the beacon lifetime. As our goal is to minimize the channel access collisions and that message 
expiration is not part of this work, in the following, we set the backoff counter to be equal to the maximum value of IEEE 802.11 protocol $(C W=1023)$.

\subsection{Idle Sense}

Idle Sense [10] aims at maximizing the throughput and providing a short-time fairness for IEEE 802.11 DCF. Instead of performing the binary exponential backoff algorithm (BEB) of IEEE 802.11 after a collision or failed transmission due to channel behavior, Idle Sense dynamically adjusts the contention window size so that the contention window size of all nodes converges in a full distributed way to similar values. It performs as follows: each host estimates $n_{i}$, the number of consecutive idle slots between two transmission attempts. According to the observed values of $n_{i}$, every maxtrans transmissions, it computes $\hat{n_{i}}$, which represents the average. Then, it uses the average value to adjust its contention window size to target value $n^{\text {target }}$ computed numerically based on the IEEE 802.11 PHY and MAC layers parameters such as the slot size and average collision size. Idle Sense is based on Additive Increase Multiplicative Decrease (AIMD) mechanism to adjust the window. The heuristic is performed as follows :

- if $\hat{n}_{i} \geq n^{\text {target }} C W \longleftarrow \alpha C W$

- if $\hat{n_{i}}<n^{\text {target }} C W \longleftarrow C W+\epsilon$

where $\alpha$ and $\epsilon$ are parameters of the AIMD algorithm. While in the first version of Idle Sense [10], maxtrans was chosen by simulation and fixed, in [9] they proposed adaptive algorithm to update the value of maxtrans in order to speed up the convergence of the average $\hat{n}_{i}$ to the target value. Idle Sense is designed for unicast data transmission, because nodes may need to detect a collision in order to adjust their contention window, while in our work, the communication is performed through broadcast transmission. As we assume that each reader can track its right neighborhood size and according to the value of slot size selected in this work and formulas (4)-(5) and (10)-(12) of [10], we provide in Table 1 for each neighborhood size its optimal contention window for the target value of 2.49 .

Table 1: Values of CW for $n_{i}^{\text {target }}=2.49$

\begin{tabular}{|c|c|}
\hline Neighbor Size & CW \\
\hline \hline 2 & 10.86 \\
4 & 22.72 \\
6 & 34.58 \\
8 & 46.44 \\
10 & 58.30 \\
\hline
\end{tabular}

\begin{tabular}{|c|c|}
\hline Neighbor Size & CW \\
\hline \hline 12 & 70.16 \\
14 & 82.02 \\
16 & 93.88 \\
18 & 105.74 \\
20 & 117.60 \\
\hline
\end{tabular}

\begin{tabular}{|c|c|}
\hline Neighbor Size & CW \\
\hline \hline 22 & 129.46 \\
24 & 141.32 \\
26 & 153.18 \\
28 & 165.04 \\
30 & 176.90 \\
\hline
\end{tabular}




\subsection{Sift}

Sift [14] is motivated by the limitation of classical CSMA-based approach in wireless sensor networks (WSNs) when several nodes with spatially-correlated data simultaneously attempt to report data to the sink node. It aims at reducing the latency and the collisions with a competitive throughput by using a suppression mechanism at MAC Layer. Instead all nodes report data, only few nodes called the first $R$ nodes report their data in a collision-free fashion, while the remaining other nodes inhibit their report. Instead of using BEB algorithm to access the channel, it uses a fixed-size contention window with a non-uniform geometrically-increasing probability distribution for picking a transmission slot in the window. This avoids the shortcoming due to the adaptive increasing of window size when collision occurs. This distribution function is given as:

$$
p_{r}=\frac{(1-\beta) \beta^{C W}}{1-\beta^{C W}} \cdot \beta^{-r}, \text { for } r=1 \ldots C W
$$

where $0<\beta<1$ is a distribution parameter. This distribution function $p_{r}$ increases exponentially with $r$. As its objective is to reduce the number of data reports by allowing only few nodes to report their data, when the transmission or collision occurred, all contending nodes select new random contention slots and repeat the same process. Although Sift is proposed with the aim of reducing the number of collisions through the suppression mechanism, collisions do still exist. But, they are drastically reduced. Our objective is to use Sift in order to allow collision-free channel access for all contending nodes.

As our goal is to improve the existing CSMA-based and in order to be compliant with ETSI EN 302 208-1 [20], we adapt them to follow its requirements, which means, that they are used in a multichannel system with the unit of backoff equal to $0.5 \mathrm{~ms}$. Note also that these approaches can be applied to any other CSMA-based reader-to-reader anti-collision in order to improve its performance.

\section{Performance Evaluation}

In order to highlight the benefit brought by these mechanisms, we implemented Idle Sense, Maximum Backoff, Sift, NFRA and GDRA approaches using WSNet [16], an event-driven simulator for large scale WSNs and fairly evaluate their performance under various network scenarios. We consider a dense RFID system where 100 to 500 readers are randomly deployed with uniform distribution on a network of dimension $4000 \mathrm{~m} \times 4000 \mathrm{~m}$. According to the reader maximum transmission power defined in [20] as 3.2 Watts EIRP, we set the reader-to-tag communication range to $10 \mathrm{~m}$ while their interference range is $1000 \mathrm{~m}$. For each scenario 100 simulations are run. Each simulation lasts 500s. The results are presented with $95 \%$ confidence intervals. Table 2 sums up all parameters. 


\begin{tabular}{|l|l|}
\hline \multicolumn{2}{|c|}{ Protocol Parameters } \\
\hline \hline Mechanism & Parameters \\
\hline NFRA & $\begin{array}{l}\text { AC Packet }=2.83 \mathrm{~ms}, \text { OC Packet }=1 \mathrm{~ms} \\
\text { OF Packet }=0.3 \mathrm{~ms}, \text { Beacon }=0.3 \mathrm{~ms} \\
\text { Slot size }=1.3 \mathrm{~ms}\end{array}$ \\
\hline GDRA & $\begin{array}{l}\text { AC Packet }=2.83 \mathrm{~ms}, \text { Beacon }=0.3 \mathrm{~ms} \\
\text { Contention Window Size }=32, \text { Slot Size }=5 \mathrm{~ms}\end{array}$ \\
\hline Idle Sense & Slot Size $=0.5 \mathrm{~ms}$, Contention Window $=$ N $/ \mathrm{A}$ \\
\hline Max. Backoff & Slot Size $=0.5 \mathrm{~ms}$, Contention Window $=1024$ \\
\hline SIFT & Slot Size $=0.5 \mathrm{~ms}$, Contention Window $=32$ \\
\hline \hline \multicolumn{2}{|c|}{ Common Parameters } \\
\hline Parameter & Value \\
\hline Reader-to-tag communication length & $0.46 \mathrm{~s}$ \\
\hline Number of tags & 800 \\
\hline \hline
\end{tabular}

Table 2: Simulation Parameters

\subsection{Performance metric}

Before presenting our simulation results, in this section, we briefly introduce our performance evaluation metrics:

- Collision: We can distinguish two types of collisions: channel access collision and reading collision. When the former occurs, we consider that the tags reading process is unsuccessful while the latter problem will just impact the throughput of tags reading. According to the transmission power used in this work, the latter will generally happen only if there is carrier-sense errors. Note that due to some of our assumptions in this work, reading collisions can not happen.

- Throughput: We define the throughput as the ratio of the average number of successful query sections per reader over the simulation duration. The higher system throughput, the more efficient protocol.

- Efficiency: It defines the ratio of the number of successful query sections over the total number of attempted query sections (i.e. successful and lost query sections).

- Jain's Fairness Index: In the literature, the fairness property is generally evaluated with the Jain's fairness index, which describes how similar and fair the resources allocated to each reader are. It is computed as follows:

$$
J=\frac{\left|\sum_{i=1}^{N} X_{i}\right|^{2}}{N \sum_{i=1}^{N} X_{i}{ }^{2}}
$$

where $\mathrm{N}$ and $X_{i}$ are respectively the number of readers in the network and the throughput of the $i$-th reader.

- Latency: It describes the average time to read all tags.

- Coverage: It defines the average number of tags read by readers during 100s of simulation. 


\section{$5.2 \quad$ Results}

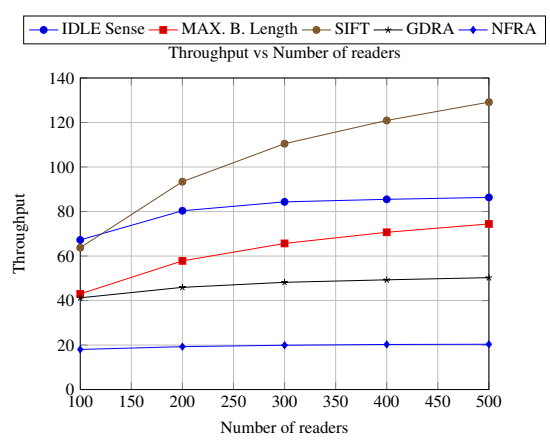

(a) Throughput vs Number of readers $\rightarrow-$ IDLE Sense $\rightarrow$ - MAX. B. Length $\rightarrow$-SIFT $\rightarrow$ GDRA $\rightarrow$-NFRA

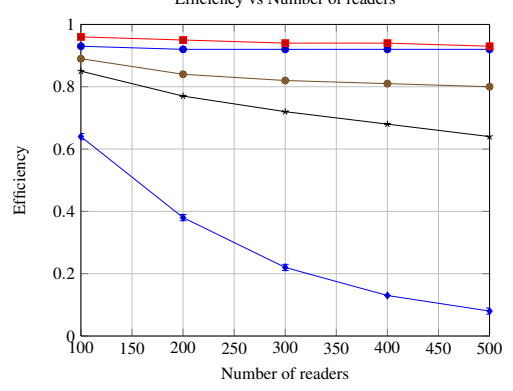

(c) Efficiency vs Number of readers

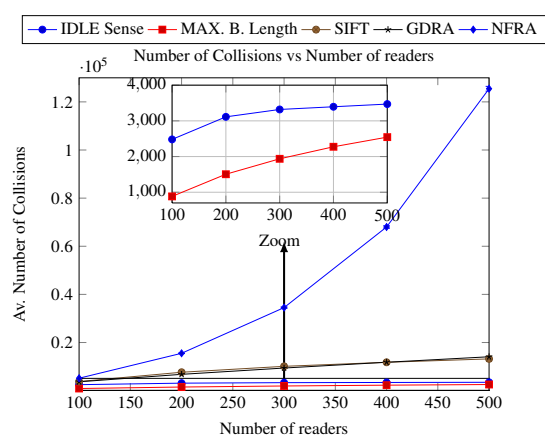

(b) Collisions vs Number of readers

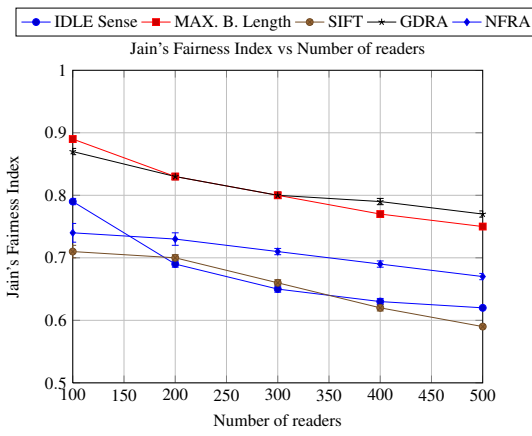

(d) Fairness vs Number of readers

Fig. 1: Performance of GDRA, Idle Sense, Maximum Backoff, NFRA and Sift schemes. (1a) Throughput, (1b) Number of collisions, (1c) Protocol efficiency and (1d) Jain's Fairness Index vs The number of readers

Fig. (1a) illustrates the throughput according to the number of readers in the system. The improvement of the proposed backoff schemes over the activity scheduling approaches is obvious. It is mainly explained by the polling process happening prior to any transmision in these protocols. However GDRA still display a higher throughput thanks to the use of the geometric distribution upon the choice of an adequate timeslot and the multichannel setting. The gap shown between the proposed backoff schemes is explained by their contention delay, the shorter it is, better the throughput is, which explains Sift the highest performances. Fig. (1b) shows the number of collisions based on the number of readers. The high number of collisions recorded by NFRA is due to the uniform distribution used for the selection of a timeslot. The use of the geometric distribution in GDRA and Sift allows them to show similar performances, not totally eliminating collisions but reducing them. Thanks to the use of a large contention 
window in Maximum Backoff, the number of collisions is highly reduced offering here the best performances. Fig. (1c) displays the efficiency according to the number of readers, it is the number of successful transmissions on the total number of transmissions attempted. The high number of collisions recorded with NFRA explains its poor performances here, while the low number of collisions observed with Maximum Backoff or Idle Sense, though they are not offering the best throughput, allow them to be the most efficient protocols. The mid-range efficiency obtained by Sift and GDRA is explained by the way they both deal with the geometric distribution to reduce the collisions. Fig. (1d) exhibits the Jain's fairness Index based on the number of readers in the system. This index allows to show how fair is the access to the transmission medium among readers. GDRA and Maximum Backoff display almost similar performances but for different reasons. The large contention window used in Maximum Backoff allows each reader to access to the channel with a fair number of collisions. Though Sift uses the same geometric distribution as GDRA does, the difference observed here is explained by the use of the polling server in GDRA which ensures in a way that readers get a fair access to the channels. The high number of collisions noticed in NFRA is also the reason why such a high drop of performance is observed as the number of readers in the system goes up.

\subsection{Coverage and Latency}

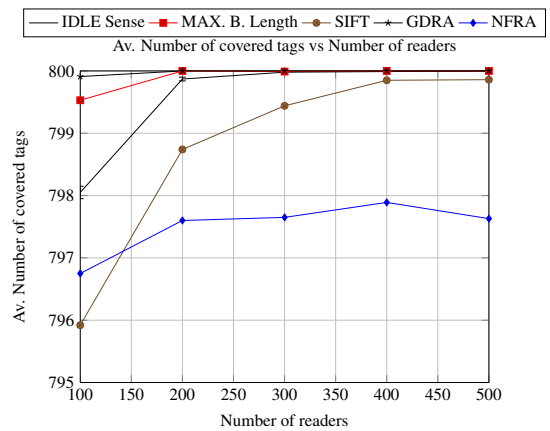

(a) Average covered tags vs Number of readers

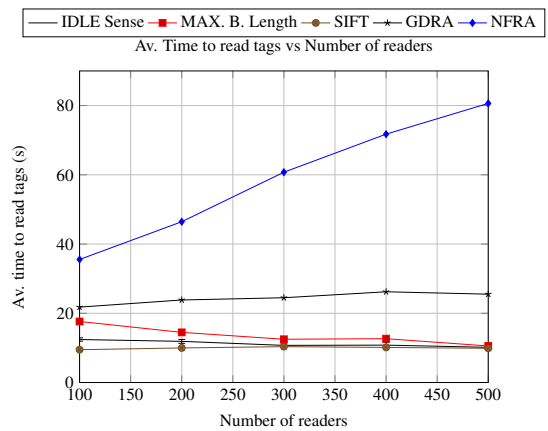

(b) Average latency vs Number of readers

Fig. 2: Performance of GDRA, Idle Sense Maximum Backoff, NFRA and Sift when 800 tags are deployed. (2a) Number of covered tags and (2b) Time necessary to cover tags vs The number of readers

Fig. (2a) illustrates the average number of covered tags according to the number of readers in the system. The high performances observed with GDRA and Maximum Backoff is due to their high fainess behavior, since access to the channels is made in a fair way then the number of covered tags is maximized. 
Other protocols display growing performances except NFRA, which presents a poor performance. This is mainly due to the use of the uniform distribution in order to select the transmission slot, which records a high number of collisions in addition to the suppression mechanism introduced by the OF message. The average being obtained with 300 readers in the system. Fig. (2b) shows the average time to read all tags in the coverage area based on the number of readers. This graph results can be explained by looking at the Fig. (1a) where Sift and Idle Sense got the best results in terms of throughput thus resulting in them obtaining the shortest time needed to read all tags. NFRA due to the collisions registered and the low throughput takes a longer time to access all tags informations. Noticing also the fact that as the number of readers increases the delay needed for Maximum Backoff decreases accordingly which is due to the state of convergence being reached faster with the high number of contending readers.

\section{Discussion and Conclusion}

Before the introduction of [19], NFRA, though it is based on unrealistic assumptions, was considered as one of the best protocols to overcome the issue of reader-to-reader collisions problem in RFID systems. The study presented in this paper shows that it is possible to improve the performance of existing CSMAbased approaches by introducing a novel backoff scheme. Indeed, we can witness the significant improvement in terms of throughput, efficiency and fairness while being able to reduce the number of collisions and shorten the delay needed for reading the tags. Though it seemed like the best performing protocol, Sift is not a viable solution in real deployments, indeed it has been presented in a interference free environment, its performances considerably dropped when an ambient noise plus interference were introduced in the simulation settings as we have shown in our previous study. The use of Idle Sense implies the use of bistatic antennas on readers, same as in GDRA, which naturally induces a hardware modification of readers. While the approach can be appropriate in case of hardware dependent study, in our future study, our aim is to go toward a novel reader-to-reader anti-collision protocol design, which can be able to perform efficiently regardless of the target reader characteristics with no additional complexity or hardware. The second approach consists of combining Idle Sense scheme with an efficient neighbor estimation algorithm. However, when the neighbor estimation error is higher than 5\%, as we have already observed in [21], Idle Sense performs poorly. While Maximum Backoff offers the best performance regarding the number of collisions and efficiency, it is also the one displaying one of the lowest throughput due to the delay needed for readers to reach a convergence state with the right contention window size. In light of the study made in this paper, we can state that the exploration of a distributed approach, making a right estimation on the size of the contention window, would offer the best solution for reducing the number of collisions while maximizing the throughput and efficiency of the readers. Based on the results shown in this paper, we assume that an approach combining both the maximized contention window size of Renverse Backoff and 
the research made by Idle Sense to reach the theoretical optimal contention window size should offer the best tradeoff. In many large-scale RFID applications, the use of mobility devices is a strong requirement, because the unbalanced link behavior can induce uncover tags or tags are attached to mobile things, that should be taken into account in the design of an efficient protocol. To deal with this challenge issue, our future research should be also oriented to mobility aspect of both readers and tags.

\section{Acknowledgments}

This work is partially supported by a grant from CPER Nord-Pas-de-Calais/FEDER Campus Intelligence Ambiante.

\section{References}

1. M.V. Bueno-Delgado and R. Ferrero and F. Gandino and P. Pavon-Marino and M. Rebaudengo. A Geometric Distribution Reader Anti-Collision Protocol for RFID Dense Reader Environments. In IEEE Transactions on Automation Science and Engineering, Vol.10:(2), pp.296-306, 2013.

2. S. Birari, S. Iyer. PULSE: a MAC protocol for RFID networks, In Proc. of EUC 2005 Workshops, 2005.

3. J.-B. Eom, S.-B. Yim, T.-J. Lee. An Efficient Reader Anticollision Algorithm in Dense RFID Networks With Mobile RFID Readers, In Trans Ind Electron, Vol. 56 (7): 2326-2336, 2009.

4. EPCglobal Standard specification. EPC TM radio-frequency identity protocols class-1 generation-2 UHF RFID protocol for communications at $860 \mathrm{Mhz}-960$ Mhz version 1.2.0, 2007.

5. R. Ferrero, F. Gandino, B. Montrucchio, M. Rebaudengo. A Fair and High Throughput Reader-to-Reader Anticollision Protocol in Dense RFID Networks, IEEE Trans. Industrial Informatics, Vol. 8(3):697-706, 2012.

6. F. Gandino, R. Ferrero, B. Montrucchio, M. Rebaudengo. DCNS: an Adaptable High Throughput RFID Reader-to-Reader Anti-collision Protocol, IEEE Trans. on Parallel and Distributed Systems, Vol. 99:1045-9219, 2012.

7. F. Gandino, R. Ferrero, B. Montrucchio, M. Rebaudengo. Probabilistic DCS: An RFID reader-to-reader anti-collision protocol, J. Network and Computer Applications, Vol. 34(3):821-832, 2011.

8. E. Hamouda, N. Mitton and D. Simplot-Ryl. Reader Anti-Collision in Dense RFID Networks With Mobile Tags, In IEEE Intern. RFID-TA, 2011.

9. Y. Grunenberger, M. Heusse, F. Rousseau, A. Duda. Experience with an implementation of the Idle Sense wireless access method, In Proc. ACM CoNEXT, pp.24:124:12, 2007.

10. M. Heusse, F. Rousseau, R. Guillier, A. Duda. Idle sense: an optimal access method for high throughput and fairness in rate diverse wireless LANs, In Proc. Conf. on Applications, Technologies, Architectures, and Protocols for Computer Communications. (SIGCOMM), pp.121-132, 2005.

11. K. Hwang, K. Kyung-Tae, E. Doo-Seop. DiCa: Distributed Tag Access with Collision-Avoidance Among Mobile RFID Readers, In Proc. EUC Workshops, 2006. 
12. D. Simplot-Ryl, I. Stojmenovic, A. Micic, A. Nayak. A hybrid randomized protocol for RFID tag identification. Sensor Review, Vol.26(2):147-154, 2006.

13. R. Stanica, E. Chaput, A.-L. Beylot. Enhancements of IEEE 802.11p Protocol for Access Control on a VANET Control Channel, In Proc. of ICC, 2011.

14. K. Jamieson, H. Balakrishnan, and Y.C. Tay Sift: a MAC protocol for eventdriven wireless sensor networks, In Proc. 3rd European Workshop on Wireless Sensor Networks, pp. 260-275, 2006.

15. J. Waldrop, D.W. Engles, S.E. Sarma. Colorwave: An anticollision algorithm for the reader collision problems, in Proc. of ICC, 2003.

16. WSNet. Wsnet:http://wsnet.gforge.inria.fr/

17. A. Lazaro, D. Girbau, and R. Villarino. Effects of interferences in UHF RFID systems, Progress In Electromagnetics Research, Vol.98:425-443, 2009.

18. D.-Y. Kim, H.-G. Yoon, B.-J. Jang, and J.-G. Yook. Interference analysis of UHF RFID systems, Progress In Electromagnetics Research B, Vol.4:115-126, 2008.

19. K. S. Leong and M. Leng Ng and P. H. Cole The reader collision problem in RFID systems, IEEE International Symposium on Microwave, Antenna, Propagation and EMC Technologies for Wireless Communications (MAPE 2005), pp. 658-661, 2005.

20. ETSI EN 302 208-1 Version 1.4.1 2011,Available online at: www.etsi.org.

21. I. Amadou and N. Mitton. Revisiting Backoff algorithms in CSMA/CA based MAC for channel Reservation in RFID reader Networks through broadcasting, In IEEE 9th International Conference on Wireless and Mobile Computing, Networking and Communications (WiMob), pp. 452-457, 2013. 